Aus der pathologischen Abteilung des kaiserl. Instituts für experimentelle Medizin in St. Petersburg.

\title{
Zur Technik der Nähte an Gefäßen kleinen Kalibers ${ }^{1}$ ).
}

\author{
Von N. A. Dobrowolskaja.
}

(Mit 7 Abbildungen im Text und 16 Figuren auf Tafel II.)

Dank den neuen Aussichten, die mit der Entwicklung und Vereinfachung der Gefäßnahttechnik sowie in der praktischen als auch in der experimentellen Chirurgie eröffnet worden sind, wird der Gefäßnaht von seiten der Chirurgie gegenwärtig eine besondere Aufmerksamkeit zuteil. Die Technik einer Nahtlegung an großen Gefäßen ist heutzutage dermaßen ausgearbeitet, daß schon viele Gefäßoperationen Eigentum der praktischen Chirurgie geworden sind (Matas, Zeidler, Wieting, Carrel und Guthrie, Bertram Bernheim, Delbet, Schak und Hesse, Ruot te u. a.). Massentransplantation verschiedener Organe haben an Menschen einstweilen wenig Anwendung gefunden (Jaboulay, Unger) und sind Gegenstand experimenteller Bearbeitung einer ganzen Anzahl von Gelehrten (Carrel, Carrel und Guthrie, Borst und Enderlein, Garrè, Stich, Stich und Makkas, Lissowskaja und viele andere). Wobei nicht selten die Kleinheit des Gefäßkalibers der zu verpflanzenden Organe große technische Schwierigkeiten bietet, was $\mathrm{Carrel}$ auch veranlaßte, sich dahin auszusprechen, da $B$ die Chancen für den Erfolg um so geringer sind, je kleiner der Durchmesser der zu vernähenden Gefäße ist.

I) Im Auszuge vorgetragen und die Präparate vorgelegt in der Ärzteversammlung des Obuchow-Hospitals am 13. April und in der Versammlung der medizinisch-biologischen Gesellschaft im medizinischen Institut für Frauen in St. Petersburg, den 24. April I9r2. 
Um die auftretenden Schwierigkeiten, wenn man es mit kleinen Gefäßen zu tun hat, zu umgehen, nahmen verschiedene Autoren, wie bekannt, die zu verpflanzenden Organe mit Stücken der großen Gefäße, in die die Gefäße des gegebenen Organs münden, und nähten sie in die entsprechenden Stellen ebenfalls großer Gefäße der Gegend, wohin das Organ verpflanzt werden sollte (Carrel, Stich und Makkas, Borst und Enderlen).

In Fällen, wobei eine Naht kleiner Gefäße nicht zu umgehen war, benutzten die meisten Autoren die von Carrel im Jahre I 902 vorgeschlagene und von $\mathrm{Stich}, \mathrm{Makkas}$ und Dormann in Details ausgearbeitete Methode der zirkulären Gefäßnaht.

Die Prothesenmethode von $\mathrm{Payr}$ und die Invaginationsmethode von Murphy sind hierbei wenig anwendbar, da sie an und für sich schon eine Verengerung des Gefäßlumens herbeiführen. Das Bemerkenswerte der Carrel-Stichschen Methode besteht, wie bekannt, darin, daß durch die ganze Dicke der Gefäßwand drei unterstützende Grundnähte gelegt werden, mit deren Hilfe das Gefäß erweitert wird, und das Lumen die Form eines Dreiecks bekommt. Die Seiten dieses Dreiecks werden der Reihe nach durch fortlaufende Naht, Intima zu Intima, miteinander vereinigt. Ein Jahr nach $\mathrm{Carrel}$ veröffentlichte Jensen eine Arbeit, worin er unter verschiedenen, zur Vereinigung der Enden eines durchgeschnittenen Gefäßes vorhandenen Verfahren der Nahtmethode den Vorzug gibt. Dabes schlägt er vor, zwecks Ausstülpung und besserer Anpassung der Intima die Grundnähte, zwei an Arterien und zwei bis drei an Venen, in U-förmigen Nähten anzulegen. Bei uns in Rußland ist die Methode Carrels durch Morosowa insofern vereinfacht worden, daß man die drei unterstützenden $C$ arrelschen Nähte durch zwei ersetzen kann.

Die Technik der Gefäßnaht wurde hauptsächlich an Hunden oder anderen ziemlich großen Tieren ausgeübt, wobei die Karotiden, Jugularvenen und Oberschenkelgefäße zum Experimentieren am zweckmäßigsten $z u$ sein scheinen. Die Arteria und Vena der Fossa axillaris sind dagegen infolge ihrer tiefen Lage weniger geeignet. - Die große, im Jahre I9I I erschienene Arbeit von $\mathrm{H}$. Yamanoüchi ,Über die zirkuläre Gefäßnaht", ist eben an diesen Gefäßen an Hunden gemacht worden. 
Entnehme aus dieser Arbeit die Maße einiger Gefäßdurchmesser: Versuch Nr. 63, großer Hund, Art. femoralis sin. $-2 \mathrm{~mm}$; Versuch Nr. I2, Hündin mittlerer Größe, Art. femoralis sin. - I,4 mm; Versuch Nr. I4, großer Hund, Art. femoralis dextra - 2,2 mm; Versuch Nr. I24, Hündin mittlerer Größe, Art. carotis comm. dextra - I,5 mm; Versuch Nr. 3, großer Hund, Art. carotis comm. dextra - $2 \mathrm{~mm}$; Versuch Nr. 2, Hund mittlerer Größe, Art. femoralis dextra - I,2 mm; Versuch Nr. 15, große Hündin, Art. carotis sinistra I,7 $\mathrm{mm}$ usw.

Aller Wahrscheinlichkeit nach hat Yamanoüchi diese Ausmessungen gleich nach der Gefäßdurchschneidung gemacht, wenn der zirkuläre Muskel infolge der erhaltenen Reizung sich im Zustande starker Kontraktion befindet, wodurch auch, wie es scheint, die an den Gefäßen großer Tiere erhaltenen niedrigen Daten zu erklären sind. Ungefähr dieselben Daten hinsichtlich der Größe des Gefäßdurchmessers werden in der Abhandlung von Stich, Makkas und Dowman angegeben.

Auf den Vorschlag von E. S. L ond on beschäftigte ich mich mit der Ausarbeitung der Nahttechnik an Gefäßen kleinen Kalibers -- an Arterien meistens unter $2 \mathrm{~mm}$ im Durchmesser und an den denselben entsprechenden Venen. Zu diesem Zweck benutzte ich $3-5$, selten $8 \mathrm{~kg}$ schwere junge und kleine erwachsene Hunde, indem ich ihnen an den Karotiden, Jugularvenen und Oberschenkelgefäßen Nähte anlegte. Wenn man bisweilen in Ermangelung kleiner Hunde gezwungen war, an großen zu experimentieren, so wurde bei ihnen zwecks Operation die Vena saphena gewählt. Da eine unmittelbare Ausmessung dieser Gefäßlumina, die nach der Durchschneidung während der Operation kaum I mm großen Durchmesser zeigen, sehr schwer genau zu machen ist, so arbeitete ich nach folgender Methode:

Während der Operation wurde keine Messung gemacht, jedoch gleich nachdem man, nach einem längeren und kürzeren Zeitraum nach der Operation dem Tier das Gefäß entnommen, wurde letzteres in der Längsrichtung durchschnitten, auf dem Finger ausgebreitet und der Umfang der gespaltenen Intima mit Hilfe eines durchsichtigen graduierten Lineals ausgemessen. Nach der erhaltenen Größe ist es nicht schwer, sich ein Urteil über den Gefäßdurchmesser zu bilden, indem man sie durch 3,14 $(\pi)$ dividiert. Aller Wahrscheinlichkeit nach sind die auf diese Art und Weise erhaltenen Daten 
etwas größer, als diejenigen nach einer unmittelbaren Ausmessung während der Operation, dafür können aber hierbei verschiedene unvorhergesehene Umstände ihren Einfluß weniger geltend machen, und außerdem ist die Möglichkeit gegeben, das Maß des Gefäßumfanges an unversehrten Stellen mit demjenigen an der Nahtlinie zu vergleichen.

Bei Anlegung einer zirkulären Gefäßnaht ist an dieser Stelle immer eine Verengerung des Gefäßlumens zu befürchten. Um so eine Verengerung zu vermeiden, empfiehlt die Methode Carrel-Stich das Gefäß mit drei unterstützenden Ligaturen so weit auseinanderzuziehen, bis das Gefäßlumen die Form eines gleichschenkligen Dreiecks bekommt. Und doch scheint mir unmöglich, zu behaupten, daß bei dieser Methode gar keine Verengerung, selbst nicht die minimalste Einkerbung der Nahtstelle, zu bekommen sei, und diese wenn auch ganz unbedeutende Verengerung wird um so gefährlicher, je kleiner das Gefäß am Kaliber ist.

Von den oben auseinandergesetzten Erwägungen ausgehend, ist es die Aufgabe dieser Arbeit, eine Methode ausfindig zu machen, nach der eine Naht derartig angelegt werden kann, daß die Möglichkeit einer zirkulären Verengerung des Gefäßes ausgeschlossen wird. Zu erreichen ist dieses durch Verlängerung der Nahtlinie mit Hilfe verschiedener Anordnung der Querschnitte und auch durch spezielle Technik der Nahtanlegung. Die einfachste Methode, wobei die Möglichkeit gegeben ist, den Einfluß der Naht nicht auf eine Stelle des Gefäßes zu konzentrieren, liegt in der Verlegung der durch die Naht durchgehenden Schnittfläche aus der steil senkrechten in eine mehr oder weniger geneigte Richtung. Dieses wird durch eine schräge Durchschneidung des Gefäßes erreicht, und je näher die Richtung des Schnittes derjenigen des Gefäßes selbst kommen wird, um so geringer wird der Einfluß der Naht auf den Durchmesser der Gefäßschnittfläche sein. Dieses Verfahren führt nur zu einer unbedeutenden Verkürzung des Gefäßes selbst, was auch bei einer quer angelegten Zirkulärnaht der Fall ist (Fig. I).

Die: Operation wurde folgendermaßen ausgeführt: nach dem gewöhnlichen Isolieren und Anlegen von Klemmen wurde das 
Gefäß mit einer zur Achse des Gefäßes unter dem Winkel von $45^{-}-35^{\circ}$ gestellten Schere auf einen Schlag durchtrennt. Nach Entfernung des überflüssigen periadventitiellen Gewebes von den Gefäßenden wurden von innen nach außen an die entferntesten Punkte des so erhaltenen ellipsoiden Querschnittes zwei durchgehende Grundnähte angelegt; zu diesem Zwecke befanden sich an jedem Faden zwei Nadeln, und beide Nähte wurden von dem Chirurgen und seinem Assistenten gleichzeitig zusammengebunden. Mit Hilfe dieser Ligaturen wurde das Gefäß fixiert und auseinandergezogen, dann wurde mit einem von den vier erhaltenen Enden eine fortlaufende Gefäßnaht angelegt. Nach Beendigung der Naht wurden die beiden Klemmen, ohne sie
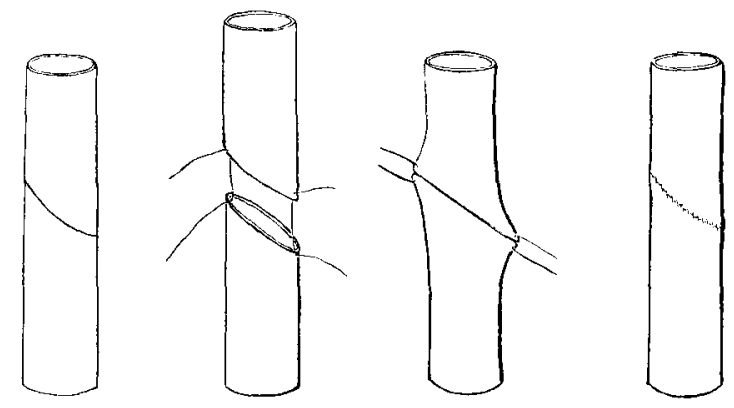

Fig. I.

vollständig zu entfernen, gleichzeitig gelockert; dabei strömt das Blut zur Stelle der Naht in zwei entgegengesetzten Richtungen und drängt, wenn Luft im Gefäße vorhanden war, dieselbe heraus. Im Notfalle wurde eine blutstillende Ergänzungsnaht angelegt, die Klemmen endgültig entfernt und die Wunde mit derselben dünnen Gefäßseide schichtweise sorgfältig verschlossen. An Welpen nähten wir auch die Haut mit einer Gefäßnadel, wodurch die Gefahr einer abermaligen Stichinfektion geringer ist, und man eine außerordentlich feine kleine Narbe erhält. Außer Kollodium wurde kein Verband gebraucht. Im Resultat der eben beschriebenen Methode erhält man eine schräge Lage der Nahtlinie, wodurch die Möglichkeit einer zirkulären Verengerung des Gefäßes ausgeschlossen ist.

Weiteren Modifikationen der bearbeitenden Methode liegt das Prinzip der künstlichen Vergrößerung des Gefäßlumens an 
der Nahtstelle zugrunde, eine Art Verlegung der Nahtlinie außerhalb der gewöhnlichen Gefäßrinne. Die zu diesem Zweck anwendbaren Schnittführungen kann man mit folgenden Namen charakterisieren: eine zackige, eine kreisförmige mit Längsein-
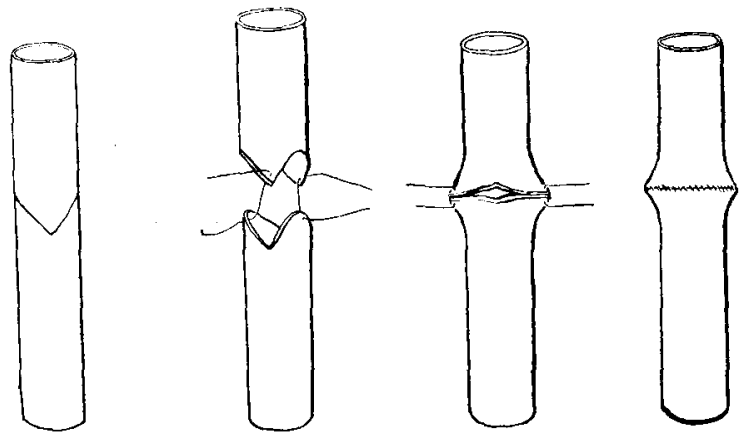

Fig. 2.

schnitten und eine läppchenförmige mit vorhergehender Anlegung einer durchgehenden fixierenden Ligatur.

Die zackige Schnittführung (Fig. 2): Nach Anlegung von Klemmen wurde der isolierte und nach Möglichkeit von
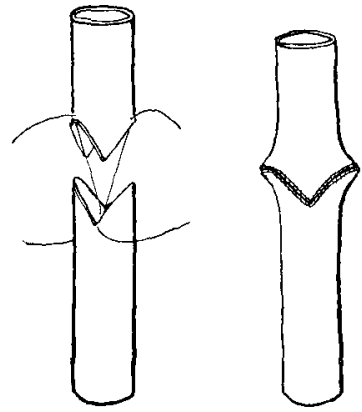

Fig. 3.

Blut befreite kollabicrte Teil des Gefäßes mit einer zur Achse des Gefäßes unter einem Winkel von $35^{-45^{\circ}}$ gestellten scharf- spitzigen Schere vom Rande bis zur Mitte schräg durchschnitten, dann wurde von der andern Seite cin dem ersten symmetrischer Schnitt gemacht, so daß beide Schnitte sich in der Mitte des Gefäßes unter einem Winkel trafen. Im Resultat einer solchen Schnittührung erhält man an beiden Enden des Gefäßes zwei gleichschenklige Dreiecke: an einem ein vorderes und hinteres, am andern ein rechtes und linkes Dreieck. Nach einer Drehung des einen Gefäßendes zum andern um 90 Grad wurden zwischen den so erhaltenen dreieckigen Läppchen an deren Basis zwei Grundnähte angelegt, an diesen Ligaturen wurde das Gefäß quer aus. einandergezogen und durch fortlaufende Naht geschlossen (Fig. 3). 
Bei dieser Schnittmethode kann man die zwei ersten Grundnähte auch an die Spitzen der Läppchen anlegen (Fig. 2), da bei vorsichtigem Auseinanderziehen das Gefäß nicht zerreißt.

Die kreisförmige Schnitführung mit Längseinschnitten (Fig. 4): Das isolierte und eingeklemmte Gefäß wurde mit einem Scherenschlag quer durchschnitten, die Enden wurden dann sorgfältig von periadventitiellem Gewebe befreit und beide Gefäßwände - die vordere und die hintere in der Längsrichtung zu gleicher Zeit auf ungefähr Gefäßdurchmessergröße angeschnitten. Dieser Moment fordert eine große Sorgfalt, da man bei der geringsten Ungenauigkeit keine analogen Läppchen bekommt. Die zwei Grundnähte wurden zwischen
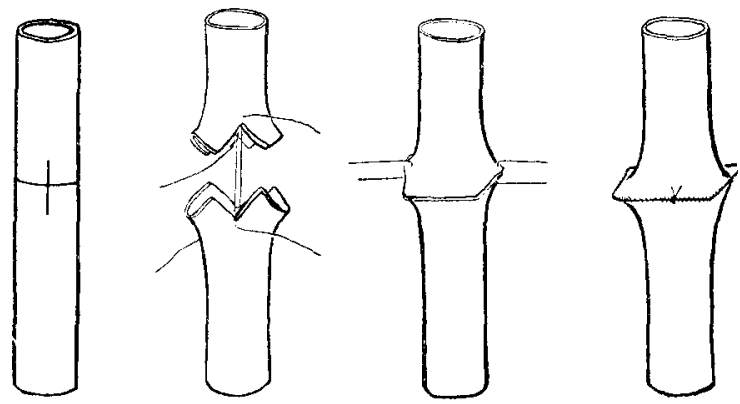

Fig. 4.

diesen beiden Läppchen an deren Basis von innen nach außen angelegt, darauf wurde das Gefäß quer in der Richtung der Lappenbasis auseinandergezogen und längs des freien Randes durch fortlaufende Naht vernäht. Die Nahtlinie gestaltet sich beim Auseinanderziehen des Gefäßes bis zum Schwinden des Lumens in ein mehr oder weniger gleichmäßiges Viereck um, deren jede Seite ungefähr die Hälfte des Gefäßumfanges gleichkommt; die Entfernung des freien Randes der aneinanderliegenden Lappen vom Gefäßzentrum erleichtert das richtige Anlegen der Naht.

Die eben erwähnte Schwierigkeit der oben beschriebenen Schnittführung veranlaßte uns, ein zuverlässigeres Verfahren zu suchen, das einem jeden, ohne eine spezielle technische Fertigkeit zu erfordern, die Möglichkeit, ein sicheres Resultat zu be- 
kommen, geben würde. Diese Nachforschungen brachten uns auf die läppchenförmige Schnittführung mit vorhergehender Anlegung einer durchgehenden fixierenden Ligatur.

Die Technik der Ausführung besteht dabei in folgendem (Fig. 5): Nach Anlegen von Klemmen wurde das Gefäß mit Hilfe einer geraden Nadel direkt in der Mitte, anfangs in der Richtung von vorn nach hinten, dann umgekehrt durchnäht, dabei wurden die Enden der Klemmen so gehoben, daß die hintere Gefäßwand zur Seite zu liegen kam. Zusammengebunden, fixierte diese Naht die vordere Gefäßwand an die hintere; dann wurden um diese Naht, wie um ein Zentrum, vier abgerundete Lappen in der Weise ausgeschnitten, daß neben der Naht nur eine ganz

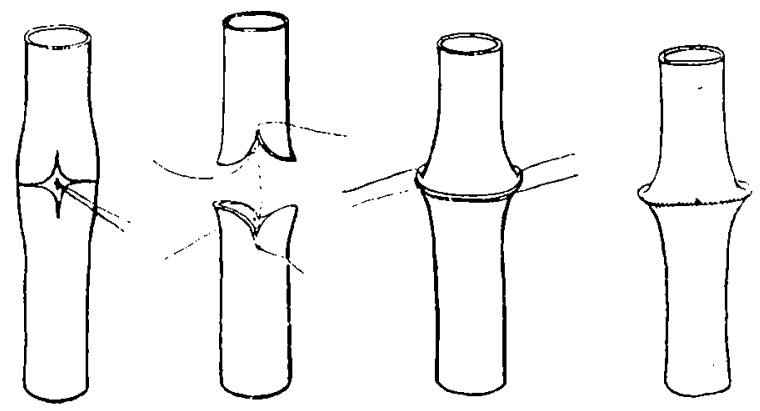

Fig. 5 .

geringe Menge Gewebes nachblieb. Die Lappen sind zu je zwei an jedem Ende des Gefäßes, einem rechten und einem linken, symmetrisch angeordnet; ihrer Größe nach unterscheiden sie sich von den in der vorhergehenden Methode erhaltenen nur durch die mit der fixierenden Naht in Wegfall kommenden Eckchen. Auch hier wurden die beiden Grundnähte in den Ecken des Schnittes an der Basis der Lappen angelegt, und das Gefäß wurde quer in der Richtung der Lappenbasis bis zum vollständigen Schwinden des Spaltes auseinandergezogen. Das Anlegen der fortlaufenden Naht wird auch hier erleichtert durch das Aneinanderliegen der Lappen und die Entfernung der freien Ränder von dem Gefäßzentrum, wodurch die Möglichkeit, die entgegengesetzte Gefäßwand zu streifen, ausgeschlossen ist. Beim Auseinanderziehen des Gefäßes durch unterstützende Liga- 
turen erhält die Nahtlinie die Form eines mehr oder weniger gleichmäßigen Kreises, dessen Durchmesser ungefähr der Hälfte des Gefäßumfanges gleichkommt.

Die eben beschriebenen zwei Lappen zu beiden Seiten kann man auch auf etwas andere Art und Weise erhalten, und zwar indem man an den Rändern des kollabierten Gefäßes zwei durchgehende fixierende Ligaturen anlegt und dann an jedem Gefäß. ende zu gleicher Zeit durch halbkreisförmige Schnitte zwei Lappen ausschneidet, so daß hierbei keine seitlichen, sondern ein vorderer und ein hinterer Lappen entstehen. Beim Ausschneiden der Lappen wird das Gefäß mit Hilfe fixierender Ligaturen, die nach dem Ausschneiden wegfallen, gehoben und leicht auseinandergezogen (Fig. 6).

Wie bei der kreisförmigen Schnittführung mit Längseinschnitten als auch bei der lappenförmigen Schnittführung ist die Anlegung von zwei cirundnähten an der Lappenbasis in deren $Z_{w}$ ischenräumen und das Auseinanderziehen des Gefäßes quer in der Richtung der Lappenbas is von außerordentlicher Wichtigkeit. Wenn man die Grund-

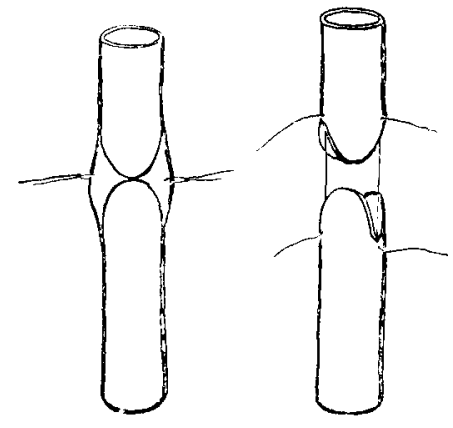

Fig. 6. nähte an die Spitze der Lappen anlegt, so reißt das Gefäß beim Anziehen unvermeidlich in die Längsrichtung, und die Verwirklichung einer regelrechten Naht wird zur Unmöglichkeit.

Also gelingt es, mit Hilfe dieser drei Schnitt. führungen, der zackigen, der kreisförmigen mit Längseinschnitten und der lappenförmigen, ein kleines Gefä $B$ scheinbar in ein größeres umzugestalten; es gelingt, auf dem Niveau der Naht einen gewissen Reserveraum zu erzeugen, der im Falle leichter Auflagerungen zelliger Blutelemente des Fibrins in der Nahtlinie das Gefäßlumen vor Verengerung schützt.

In dieser Art erscheint die von uns angewandte Methode der Naht an Arterien. Venen kleinen Kalibers haben die Eigentüm. 
lichkeit, daß infolge der Feinheit und Welkheit ihrer Wände das Gefäßlumen nach der Durchschneidung dermaßen kollabiert und verklebt, daß3 es bisweilen außerordentlich schwerfällt, das selbe zu finden und mit einer Beschädigung des Gefäßes durch Pinzetten verbunden ist, was eine Verzögerung der Operation herbeiführt usw. Außerdem kommt es an Venen viel schneller als an Arterien durch die Naht zu Verengerungen des Gefäßlumens, da die dünne nachgiebige Gefä $\beta$ wand beim Zusammenziehen einzelner Stiche der fortlaufenden Naht sich leicht in Falten legt. In dieser Hinsicht könnte die Knopfnaht einige Vorteile bieten, jedoch ist sie, besonders an kleinen Gefäßen, dermaßen kompliziert und schwer, daß es sehr erwünscht wäre,
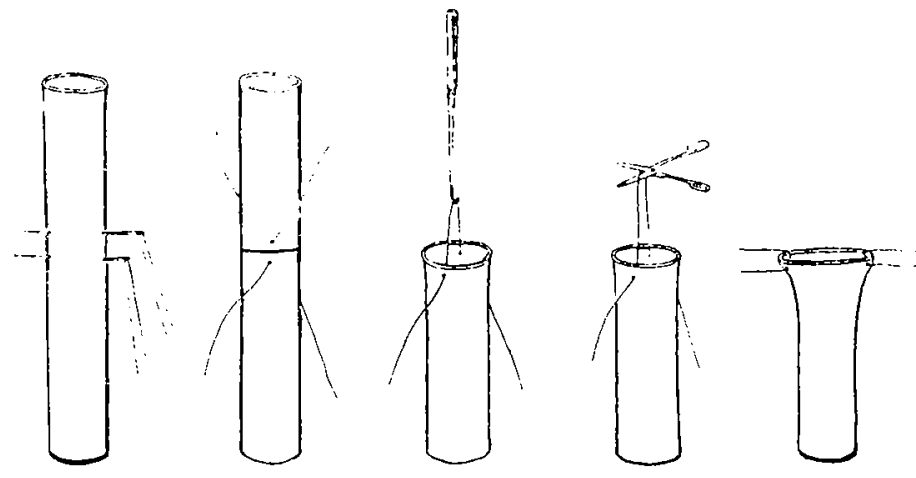

Fig. 7 .

sie zu umgehen. Als Handgriff zum leichteren Auffinden des GefäBlumens und späterhin auch zum Auseinanderziehen desselben bis zu seiner maximalen Weite kann die Durchführung vorausgehender vorläufiger, temporärer fixierender Durchgangsligaturenfäden dienen. Um die maximale Erweiterung des Gefäßes durch den Blutdruck zu erhalten, wurden auf eine isolierte Vene, anfangs auf ihr zentrales, dann auf das periphere Ende, je eine Klemme angelegt - an großen Gefäßen ist es bequemer, in kollabiertem Zustande zu arbeiten. Indem man dann die Fnden der Klemmen scheitelrecht hob, machte man die hintere Gefäß3wand zugänglich und führte in der Mitte von hinten nach vorn durch das Gefäß cine gerade Gefäßnadel mit einem ziemlich langen Seidenfädchen durch; eine zweite ebensolche Nadel mit Faden wurde parallel dieser auf $\mathrm{I}^{1} / \mathrm{2}-2 \mathrm{~mm}$ tiefer durchgeführt. 
Das Gefäß wurde mit der ersten Nadel nur durchstochen, der Faden aber bis zur Durchführung der zweiten Nadel nicht durchgezogen, damit das Blut nicht vorzeitig ausfließt und die Spannung der Gefäßwand nicht nachläßt, wodurch die richtige Durchführung der Ligaturen erleichtert wird. Nachdem die Fäden durchgeführt und der eine Faden nach oben, der andere nach unten umgebogen waren, wurde das Gefäß zwischen ihnen in querer oder schräger Richtung durchschnitten. Daraufhin irgendeine Stelle des Gefäßrandes mit der Pinzette fassend, drang man mit einem einfachen dünnen Häkelhaken in das Lumen, faßte damit den durch das Lumen gehenden Teil des Fadens, zog ihn hervor und durchschnitt ihn. Man bekam hiermit zwei durchgehende Ligaturenfäden, mit deren Hilfe das Gefäß auseinandergezogen, das Lumen geöffnet und die Intima sogar leicht ausgestülpt wurden. Dasselbe wurde auch mit dem andern Gefäßende gemacht. Den Rand des Gefäßes jetzt vorsichtig mit der Pinzette hinter das periadventitielle Gewebe zurückziehend, legten wir in senkrechter Richtung zu den durchgehenden fixierenden Ligaturenfäden zwei Grundnähte an und, indem man abwechselnd die Grundnähte und die fixierenden Ligaturenschlingen anzog und andauernd das Gefäß erweiterte, machte man, ohne die Gefäßränder mit der Pinzette $\mathrm{zu}$ berühren, eine fortlaufende Randnaht.

Die Anwendung durchgehender fixierender Ligaturen kann man den verschiedenen Schnittformen anpassen; die Details dieser Methode bearbeiten wir. Wie bei allen Gefäßnahtmodifikationen an Arterien, so bleibt auch hinsichtlich der Venen alles Obengenannte bezüglich Blutstillung, Vernähung der weichen Gewebe über dem Gefäß, Hautverschluß usw. usw. in Kraft.

Die oben beschriebenen Schnittformen, die bei allen möglichen chirurgischen Operationen, besonders in der Chirurgie der Extremitäten, große Anwendung finden, wurden hinsichtlich der Gefäße vorerst noch sehr wenig verwandt. So wird bei End-zuSeitanastomosen zweier parallel gehender Gefäße das zu verpflanzende Gefäß gewöhnlich schräg durchschnitten zwecks besseren Anpassens zum Spalt des Gefäßes, in das es verpflanzt wird (bei der Operation von Delbet).

In den Versuchsprotokollen von $\mathrm{Yamanoüchi} \mathrm{findet} \mathrm{sich}$ 
eine Angabe, daß er einmal mit Erfolg eine schräg durchschnittene Vene vernähte. Hinsichtlich der Anwendung an Gefäßen der von uns beschriebenen kreisförmigen Schnittführung mit Längseinschnitten fand ich in der Literatur nur eine Arbeit von Pirovano. Die nach dieser Schnittführung erhaltenen viereckigen Lappen verbindet er paarweise, und die Zwischenräume verschließt er durch fortlaufende Randnaht. Irgendwelche Angaben bezüglich Anwendung anderer Arten von Schnitten an Gefäßen und ebenso die Methode durchgehender fixierender Ligaturenfäden ist mir in der Literatur nicht möglich zu finden gewesen.

Um mit der Beschreibung der Methodik zu schließen, will ich noch beiläufig einige Worte über das von uns gebrauchte Instrumentarium sagen. Kleine leichte $\mathrm{D}$ i ef f e $\mathrm{n}$ b a ch sche Klemmen wurden an Arterien unmittelbar angelegt, an Venen jedoch, wo sie eine bedeutende Verklebung der Gefäßwände hervorrufen können, wurden ihre Enden vor der Anlegung mit einer dünnen Schicht Watte umwickelt, oder man faßte zu gleicher Zeit einen Teil des um das Gefäß befindlichen Zellgewebes mit. Zur Ausführung korrekter Schnitte ist eine gute Schere mit starken scharfen Spitzen selbstverständlich das Wichtigste; für den Lappenschnitt ist eine spezielle Schere mit starker Flächenkrümmung ganz an der Spitze erwünscht. Zu nähen ist, wie bekannt, leichter und schneller mit geraden Nadeln ohne Nadelhalter; jedoch in Fällen von Anlegung einer Gefäßnaht in der Tiefe, z. B. an Gefäßen der Niere, ist man gezwungen, gebogene Nadeln zu gebrauchen.

Als Nahtmaterial wurden Seide oo und Menschenhaar gebraucht. Letzteres hat einige Vorzüge: es ist sehr fein, genügend stark, verwickelt sich nicht während der Operation, wird von Blut nicht durchtränkt und läßt sich in das kleinste Nadelöhr leicht einfädeln. Das Menschenhaar als Nahtmaterial wird von Guthrie und Sofoterow empfohlen, letzterer weist in seiner Dissertation darauf hin, daß an Präparaten ,,das Haar fast ohne Reaktion vonseiten der Gefäßwand in dieser liegt". Zur völligen Beurteilung dieser Frage sind jedoch noch weitere Beobachtungen erforderlich. Die Sterilisation der Seide wurde durch Erhitzen bis I $5^{\circ}$ in Vaselinöl erzielt, des Haares - ebenso, doch erst 
nach vorhergehender Reinigung mit Äther und Alkohol. Man vermied es, das Gefäß mit Marli auszutupfen, sondern man durchspülte es einfach von Zeit zu Zeit mit Lockscher Flüssigkeit, was auch das Epithel vor Austrocknen schützte.

Protokolle der ersten I 5 Versuche führe ich nicht an, da diese Versuche als Ausarbeitung zur Grundlage der technischen Handgriffe dienten und natürlich kraft der im Anfange unvollkommenen Methodik eine große Zahl von Mißerfolgen zu verzeichnen haben. Da wir es außerdem manchmal mit ganz kleinen Welpen zu tun hatten, gingen uns einige davon anscheinend direkt infolge von Narkose ein. Jetzt operieren wir größtenteils nur mit Hilfe von Morphium.

Erwähnen wir noch kurz eine Reihe von Versuchen, 20 an der Zahl, an Nierengefäßen ausgeführt, da es im großen ganzen auch Operationen an kleinen Gefäßen waren. Die Idee dieser eben genannten Operation wurde schon von E. S. Lond on im Handbuch der biochemischen Arbeitsmethoden mitgeteilt und besteht im wesentlichen darin, daß zwecks Erhaltung aus dem Blute der V. portae der in dem Darmtraktus eingesaugten Produkte, eine Anastomose des zentralen Endes der V. lienalis mit dem peripheren Ende der Art. renalis gemacht wird. Um den Druck in V. portae zu erhöhen und ihr Blut in die Niere zu dirigieren, machten wir an ihr oberhalb der Mündungsstelle der $V$. lienalis mit dicker Seidenligatur eine Umschnürung. Da gewöhnlich die $V$. lienalis breiter als die Art. renalis ist, so wurde letztere in schräger Richtung durchschnitten, um ihr Lumen an Größe demjenigen der V. lienalis anzupassen. Zwei Grundnähte und die zwischen ihnen angelegte fortlaufende Naht gaben vorläufig vollkommen befriedigende Resultate. Jedoch ist es uns noch nicht gelungen, einen dauernden Erfolg zu erziclen.

Bei den meisten Hunden wurde nach Anastomose der Art. renalis mit $V$. lienalis an der rechten Niere die linke Niere entfernt; all diese Tiere kamen im Laufe der ersten $5 \times 24$ Stunden an Zeichen der Urämie um. In der kollabierten Harnblasc gelang es manchmal, 20--25 ccm trüber, an Eiweiß und Zellelementen reicher Flüssigkeit zu finden. In zwei Fällen, wobei die zweite Niere gelassen wurde, blieben die Tiere am Leben, der Harn wurde allmählich normal, jedoch nach Entfernung der gesunden Niere starben beide Hunde unter urämischen Erscheinungen.

Bei der Sektion erschien die gebliebene Niere geschrumpft und derb, die Anastomosenstelle stark verengt, die V. lienalis verdickt, dem Äußern nach der Art. renalis, als dessen Anfang sie jetzt gilt, ähnlich. 
In einem Falle entfernten wir vorerst bei einem gesunden Hunde die linke Niere und machten $3^{1 / 2}$ Monate darauf an der gebliebenen rechten Niere die oben beschriebene Gefäßanastomose. Der Hund ging ein, ein lockerer Thrombus verstopfte die Anastomosenstelle. In der Annahme, daß ein breiteres Venengefälle vielleicht für die Aufrahme des unter geringem Drucke einfließenden Blutes mehr geeignet sein würde, veränderten wir in der Niere eines Hundes die Richtung des Blutstroms und machten eine doppelte Anastomose und zwar: das zentrale Ende der $V$. lienalis vereinigten wir mit dem peripheren der V. renalis, wodurch dieses erhaltene Gefäß die Funktionen einer Arterie übernehmen sollte, dann wurde das periphere Ende der Art. renalis, dessen zentrales Ende vorerst unterbunden wurde, mit dem zentralen Ende der V. renalis verbunden, durch dieses Gefäß mußte das Blut von der Niere abfließen. Die zweite Niere ward entfernt. Der Hund lebte $2^{1 / 2}$ Tage; bei der Sektion sind die Gefäße durchgängig, die Niere stark vergrößert, dunkel, nekrotisch. In der Blase $25 \mathrm{ccm}$ Urin, mit einer Menge Zellelementen, Zylindern und Eiweiß.

Von dem technischen Gesichtspunkte der Gefäßanastomose aus bieten alle diese Operationen große Schwierigkeiten, da man, trotz des die Linea alba ein wenig überschreitenden Quer- oder Eckschnittes, doch in der Tiefe an sich mit der Atmung andauernd bewegenden Gefäßen zu arbeiten hat. Die Stelle der Anastomose war bei der Sektion mal vollständig frei, mal durch einen mehr oder weniger lockeren Thrombus verschlossen, das Auseinandergehen der Nahtlinie wurde fast nie beobachtet. Die Operation der Endenanastomose an $\mathrm{V}$. lienalis und Art. renalis ist im Jahre 1905 von Carrel und Guthrie ausgeführt worden, leider teilen sie nicht die Resultate mit.

Jetzt werde ich Protokolle der an Welpen und kleinen Hündchen ausgeführten Versuche späterer Zeit anführen; bei ihnen wurde die Naht an die Karotiden, die äußeren Jugularvenen und die Oberschenkelgefäße angelegt. Hierher gehören auch einige Versuche der Vena saphena an mehr oder weniger großen Hunden.

Welpe, $3,5 \mathrm{~kg}$ schwer. Versuch Nr. 36. Art. carot. dextr. 9. XII. I9I I - kreisförmige Schnittführung mit Längseinschnitten, mit Seide vernäht, gute Blutfüllung, pulsiert. II. I. I912 - das GefäB läßt sich leicht aus dem umgebenden Gewebe los. lösen, keinerlei Verwachsungen, die Nahtstelle gar nicht verdickt, die Naht ist von außen mit bloßem Auge zu sehen. Das Gefäß längs durchschnitten, zeigt überall ganz glatte Intima, auf dem Niveau der Naht an der Stelle der vernähten zwei Lappen eine leichte 
Eindellung, die auch mit glatter Intima belegt ist. Der Umfang des Gefäßes nach der ausgebreiteten Intima gemessen $6 \mathrm{~mm}$, an der Nahtstelle $7,5 \mathrm{~mm}$.

Versuch Nr. 37. Art. carotis sin. 9. XII. IgII zackiger Schnitt, Seidennaht, leichte Blutung. II. I. I9I2 -.. die Arterie ist verdickt und mit dem umgebenden Gewebe verwachsen, Thrombus,

Versuch Nr. 38. Art. femoralis sin. 9. XII. I9IIschräger Schnitt, starkes Auseinanderweichen der Enden, es war sehr schwer, mit gerader Nadel zu nähen. II. I. I9I2 - die Arterie ist thrombosiert.

Kleiner Welpe, gegen $3 \mathrm{~kg} \mathrm{schwer.} \mathrm{Versuch} \mathrm{Nr.} 39$. Art. carotis sin. 30. XII. I9I I - zackiger Schnitt, mit Seide genäht. 2. I. I912 - das Gefäß ist durchgängig, an der Nahtstelle unbedeutende Ablagerungen von Fibrin. Umfang des Gefäßes $5 \mathrm{~mm}$, an der Nahtstelle $6 \mathrm{~mm}$.

Versuch Nr. 40. Art. carotis dextr. 30. XII. I9II kreisförmige Schnittführung mit Längseinschnitten, mit Seide vernäht, unbedeutende Blutung, pulsiert. 2. I. I9I2 - von seiten der Naht alles in Ordnung, Umfang des Gefäßes $5 \mathrm{~mm}$, an der Nahtstelle $7 \mathrm{~mm}$.

Versuch Nr. 4I. Art. femoralis sin. 30. XII. I9rIschräger Schnitt, mit Seide vernäht, gute Blutfüllung, fast keine Biutung, pulsiert. 2. I. I912 - Arterie ist durchgängig, Umfang des Gefäßes überall 4,5 mm. Der Welpe ist infolge einer zufälligen Ursache am vierten Tage nach der Operation gestorben.

Kleines, junges Hündchen, $4 \mathrm{~kg} \mathrm{schwer.} \mathrm{Ver-}$ such Nr. 42. Art. carotis sin. 6. II. I9I2 - zackiger Schnitt, Seidennaht, pulsiert, blutet fast gar nicht. I. V. I912 - Arterie ist von Verwachsungen vollständig frei, die Naht ist schon von außen zu sehen, die Stelle der Naht gar nicht verdickt. Bei der Durchschneidung in der Längsrichtung ist die Arterie vollständig durchgängig, überall mit glatter, glänzender Intima bedeckt, durch die die Nahtstelle leicht durchschimmert. Der Umfang des Gefäßes überall $6 \mathrm{~mm}$, so daß die in der ersten Zeit nach der Operation beobachtete Verbreiterung des Gefäßes hier schon nicht zu finden ist.

Versuch Nr. 43. Art. carotis dextr. 6. II. I9I2 kreisförmige Schnittführung mit Längseinschnitten, Seidennaht, pulsiert. I. V. I9I2 - weder Verwachsungen, noch Verdickungen auf dem Niveau der Naht. Irgendwelche Vertiefungen an Stelle der vernähten Lappen, die kurze Zeit nach der Operation beobachtet wurden, sind hier nicht vorhanden; die Naht schimmert leicht durch die glänzende, glatte Intima durch. Der Umfang des Gefäßes überall $6 \mathrm{~mm}$.

Ziemlich groBer Welpe, $5,5 \mathrm{~kg} \mathrm{schwer.} \mathrm{Versuch}$ 
Nr. 44. V. jugularis extr. sin. 20. II. I9I2 - kreisförmige Schnittführung mit Längseinschnitten, da die Vene ausgleitete, war der Schnitt schwer zu machen gewesen, er ist nicht ganz kunstgerecht ausgekommen; Naht mit Seide. 7. III. 1912 - die Vene ist obliteriert.

Versuch Nr. 45. Art. femoralis dextr. 20. II. I9r2 zackiger Schnitt, Naht mit Seide, ziemlich starke Blutung, Ergänzungsnaht durch die Adventitia. 13. VI. I9I 2 - Wandthrombus, das Gefäß durchgängig.

Versuch Nr. 46. Art. carotis sin. 7. III. I9I2 zackiger Schnitt, Seidennaht. Noch unter Beobachtung.

Versuch Nr. 47. Art. carotis dextr. 7. III. I9I2 schräger Schnitt, Seidennaht. I3. VI. Thrombus.

$\mathrm{K}$ leines weiBes $\mathrm{H}$ ündchen, $4 \mathrm{~kg}$ schwer. Versuch Nr. 48. V. jugularis extr. sin. 17. III. I912 - kreisförmige Schnittführung mit Längseinschnitten, Seidennaht. 7. V. I9I2 Thrombus.

Versuch Nr. 49. Art. carotis sin. 17. III. I912. kreisförmige Schnittführung mit Längseinschnitten, Seidennaht. $7 . \mathrm{V}$. I9I2 - das Gefäß thrombosiert.

Versuch Nr. 50. V. jugularis ext. dextr. I7. III. I9I2 - schräger Schnitt, Seidennaht, nicht die geringste Verengerung. 7. V. I9I2 - die Nahtlinie ist kaum zu seheri, mit glänzender Intima bedeckt. Breite des Gefäßes überall $9 \mathrm{~mm}$.

Versuch Nr. 5I. Art. femoralis sin. 3I. III. I9I2schräger Schnitt, Seidennaht. 7. V. I9I 2 - die Nahtstelle nur leicht verdickt, Intima überall glatt, Umfang des Gefäßes überall $6 \mathrm{~mm}$.

Versuch Nr. 52. V. femoralis dextr. 3I. III. I912 schräger Schnitt, Seidennaht. 7. V. I9I2 - die Nahtstelle durch die Narbe bedeutend verengt, jedoch durchgängig und mit glänzender Intima bedeckt, die Venenwand an dieser Stelle verdickt.

Kleines weiBes $\mathrm{H}$ ündchen, schwarze Ohren, $3,5 \mathrm{~kg}$ schwer. Versuch Nr. 53. Art. carotis sin. 17. IV. 1912kreisförmige Schnittführung mit Längseinschnitten, infolge einer Blutung eine Ergänzungsnaht, pulsiert, während der Operation das Gefäß nicht breiter als $1 \mathrm{~mm}$ im Durchmesser. 24. V. - nicht die geringsten Verwachsungen mit den umliegenden Geweben, die Nahtstelle nicht verdickt, die Naht von außen gut zu sehen. Das Gefäß in der Längsrichtung durchschnitten, zeigt überall eine glatte Intima. Der Umfang des Gefäßes $5 \mathrm{~mm}$, an der Nahtstelle $8 \mathrm{~mm}$.

Versuch Nr. 54. Art. carotis dextr. I7. IV. I9I2 zackiger Schnitt, Haarnaht, pulsiert. 24. V. 1912 - die Arterie löst sich leicht von der Umgebung; die Nahtstelle selbst verengt und nicht durchgängig, jedoch bei geringstem Druck mit der Schere gibt die dünne Membran nach und es gelingt, das Gefäß in der Längs- 
richtung zu durchschneiden. Gleich zeigt sich zu beiden Seiten der Naht vollständig glatte Intima. Umfang des Gefäßes $5 \mathrm{~mm}$.

$\mathrm{Schwarzes} \mathrm{kleines} H$ ündchen, $7,5 \mathrm{~kg}$ schwer. Versuch Nr. 55. V. f emoralis dextra. 20. IV. I9I2 - schräger Schnitt mit zeitlicher Anlegung von vier durchgehenden fixierenden Ligaturen, mit deren Hilfe das Gefäßlumen an beiden Enden in Vierecke umgestaltet und durch fortlaufende Haarnaht vernäht wird. Während der Operation ist die Vene vom Ol. vaselini dichter geworden; nach Entfernen der Klemmen füllte sich das Gefäß mit Blut und nahm normales Aussehen an. Noch unter Beobachtung.

Versuch Nr. 56. Art. carotis dextr. 8. V. I9I2 Lappenschnitt mit zeitlicher Anlegung einer durchgehenden fixierenden Ligatur, Haarnaht, ziemlich starke Blutung ist durch eine ergänzende Seidennaht gestillt worden. Pulsiert.

Versuch Nr. 57. Vena jugularisexternadextr. 8. V. I9I 2 -- schräger Schnitt, mit zeitlicher Anlegung zweier durchgehender fixierender Ligaturen, Naht mit doppelt zusammengelegtem dünem, weißem Haar. Nach Entfernung der Klemmen füllte sich das Gefäß sofort mit Blut; eine Verengerung war nicht zu bemerken. Unter Beobachtung.

Versuch Nr. 58. Art. c arotis sin. 22. V. I9I2 - Lappenschnitt mit zwei seitlichen fixierenden Ligaturen. Seidennaht, die Blutung ist durch eine Ergänzungsnaht zum Stehen gebracht worden. Pulsiert. Unter Beobachtung.

Versuch Nr. 59. V. jugularis ext. sin. 22. V. I9I2 querer Schnitt mit zeitlicher Anlegung zweier durchgehender fixierender Ligaturen; Seidennaht bei starker Auseinanderziehung der Vene. Das Gefäß füllte sich bis zur normalen Größe mit Blut, keine Verengerung, unbedcutende Blutung. Unter Beobachtung.

Welpe - ganz klein, gegen $3 \mathrm{~kg}$ schwer. Versuch Nr. 6o. Art. carotis dextr. r. V. 1912 - halbkreisförmige Lappen mit zeitlicher Anlegung einer zentralen durchgehenden fixierenden Ligatur. Haarnaht. 4. VI. I912 - gar keine Verwachsungen mit den umliegenden Organen, die Nahtstelle mit bloßem Auge zu sehell und kaum fühlbar, Intima glatt, die Naht als dunkler Streifen klar zu sehen. Umfang des Gefäßes gegen $4 \mathrm{~mm}$, an der Nahtstelle $5 \mathrm{~mm}$.

Versuch Nr. 6I. Art. carotis sin. I. V. 1912 - dasselbe Verfahren wie am vorhergehenden GefäB. 4. VI. Igı 2 - gar keine Verwachsungen, Intima glatt, die Naht mit bloßem Auge zu sehen, hat die Form einer nicht scharf ausgeprägten Zacke. Umfang des Gefäßes $4 \mathrm{~mm}$, an der Nahtstelle $5,5 \mathrm{~mm}$.

Versuch Nr. 62. V. femoralis dextr. 24. V. I9I2 querer Schnitt mit Anlegung zweier durchgehender fixierender Ligaturen. Seidennaht, gute Blutfüllung. 4. VI. I912 - das Gefäß an- 
scheinend durchgängig, obwohl ein ziemlich langer Wandthrombus vorhanden ist. Umfang des Gefäßes an unversehrter Stelle 3,5 $\mathrm{mm}$.

Versuch Nr. 63. Art. femoralis sin. 24. V. $1912-$ schräger Schnitt, Haarnaht. 4. VI. I912 - mächtige Blutung, das Tier ging ein. Es war keine Möglichkeit, sich in den Geweben zurechtzufinden, die Ursache der Blutung ist unklar.

WeiBer Hund, $8 \mathrm{~kg}$ schwer. Versuch Nr. 64. V. femoralis sin. 5. V. I9I2 - querer Schnitt mit zwei durchgehenden fixierenden Ligaturen; der Versuch, das Gefäß mit einem Haar zu vernähen, mißlang, die Gefäßenden gingen stark auseinander und trotz der fixierenden Ligaturen gelang es nicht, sie mit dem Haar zusammenzubringen, das Haar riß. Das Gefäß wird mit Seide vernäht. II. V. 1912 - Eiterung, Vene in ihrem halben Umfange verheilt, im übrigen auseinandergewichen.

Versuch Nr. 65. Art. femoralis dextr. 7. V. 1912 Lappenschnitt mit zeitlicher Anlegung einer durchgehenden fixierenden Ligatur, Haarnaht, die Blutung ist durch eine Ergänzungshaarnaht gestillt worden. Das Gefäß pulsiert gut. Es gelang nicht, die Arterie mit Zellgewebe zu bedecken.

Versuch Nr. 66. V. femoralis dextr. Nach der soeben ausgeführten Operation wurde auf derselben Seite zwischen zwei durchgehenden fixierenden Ligaturen die Vene in schräger Richtung durchschnitten und mit doppelt genommenem Haar vernäht; nach Entfernen der Klemmen füllte sich das Gefäß gut mit Blut, gar keine Verengerung. II. V. 1912 - der Hund ist infolge starker Blutung eingegangen, alle Gewebe in der Inguinalgegend sind von Blut durchtränkt, man kann sich gar nicht zurechtfinden. Möglicherweise stellt das Operieren an zwei nebeneinander liegenden Gefäßen und die dadurch entstehende Verzögerung der Operation eine Gefahr vor.

$\mathrm{Schwarzes,}$ glattes Hündchen, $8 \mathrm{~kg}$ schwer. Versuch Nr. 67. V. femoralis dextr. I6. V. I9I2 - querer Schnitt mit temporärer Anlegung zweier durchgehender fixierender Ligaturfäden. Seidennaht, nach Entfernen der Klemmen hat sich die Vene gut ausgedehnt. 20. VI. I9I2 - die Vene läßt sich aus dem umgebenden Gewebe leicht herausheben und in der Längsrichtung durchschneiden, die Nahtlinie schimmert gut durch die Intima durch, die Nahtstelle auf Berührung leicht verdickt. Der Umfang des Gefäßes $8 \mathrm{~mm}$, an der Nahtstelle $7 \mathrm{~mm}$.

Versuch Nr. 68. V.f emoralis sin. 16. V. 1912 - Lappenschnitt mit zeitlicher Anlegung einer durchgehenden fixierenden Ligatur. Nach Entfernen der Klemmen ist an der Nahtstelle eine leichte Verengerung $\mathrm{zu}$ bemerken, die selbst bei künstlicher Blutdurchtreibung nicht verschwindet. Seidennaht. 20. VI. I9I2 das Gefäß ist mit einiger Mühe aus der Umgebung gelöst worden, läßt sich in der Längsrichtung leicht durchschneiden, obwohl es 
durch ein ohne Schwierigkeiten von den Wänden abgegangenes, lockeres, rotes Gerinnsel verstopft war. Die Nahtlinic hat eine leicht gewellte Form, Umfang des Gefäßes überall $8 \mathrm{~mm}$.

Versuch Nr. 69. Art. carotis sin. 3r. V. I9I2 … Lappenschnitt mit zwei fixierenden Ligaturen, mit Ilaar vernäht, Blutung mit Ergänzungsnaht gestillt, pulsiert. 20. VI. I9I2 - großer Wandthrombus, das Gefäß jedoch von einer Seite durchgängig. Umfang des Gefäßes an normaler Stelle $5 \mathrm{~mm}$.

Versuch Nr. 70. V. jugularis ext. sin. 3r. V. I912 schräger Schnitt mit zwei zeitlichen fixierenden Ligaturen; Haarnaht, blutet fast gar nicht, hat sich gut ausgredehnt. 20. VI. I912 - wird aus den sie umgebenden Teilen leicht gelöst, nicht die mindeste Verdickung an der Nahtstelle; läßt sich in der Längsrichtung vollständig leicht durchschneiden, die Nahtlinie schimmert deutlich durch die Intima durch. Zwei kleine lockere, dem Äußeren nach vollständig frische Gerinnsel beginnen sich an den Härchen der Naht zu bilden und dringen in die seitlichen feinen Ästchen ein. Limfang des Gefäßes überall o $\mathrm{mm}$.

$\mathrm{Schwarzer} \mathrm{H}$ und, gegen $8 \mathrm{~kg}$ schwer. Versuch Nr, $7 \mathrm{I}$. V. femoralis dextr. 29. T. I9I2 - schrägcr Schnitt mit zwei durchgehenden fixierenderi Ligaturfiiden, Seidennaht. Genügende Blutfüllung. Cnter Bcobachtung.

Versuch Nr. 72. V.femoralis sin. 29. V. I912 -- Querschnitt, Naht nach Carrel - drei Grundnähte, davon eine IIaarnaht, mit IIaar vernäht. Unter Beobachtung.

GroBerbunter Hund. Versuch Nr. 73. V. saphena sin. 5. VI. I9I2 - schräger Schnitt mit zwei temporären durchgehenden fixierenden Ligaturfäden. Fs hat sich gezeigt, daß der obere Abschnitt überdreht war, die Nahtstelle ist nicht verengt, es hat fast nicht geblutet. I) urch Anlegung einiger Nähte an das periadventitielle Gewebe gelang es, das Gefäl3 etwas zu glätten. Hat sich ziemlich gut mit Blut gefüllt. Unter Beobachtung.

Versuch Nr. 74. V. saphena sin. 5. VI. I9l2 … schräger Schnitt mit zwei durchgehenden fixicrenden Ligaturfäden, mit Seide vernäht, indem von jeder Seite ungefähr I $\mathrm{mm}$ Gewebe mitgefaßt wurde. Nach Entfernen der Klemmen gute Blutfüllung, blutet fast nicht. Bei künstlicher Blutdrucksteigerung im GefäB, durch $\mathrm{Zu}$ drücken des zentralen Endes, auch keine Blutung. Unter Beobachtung.

Ziemlich groBer weiBer Hund. Versuch Nr. 75. V. saphena dextr. 7.VI. I912 - schräger Schnitt mit zwei durchgehenden fixierenden Ligaturfäden, Seidennaht. Keine Verengerung, jedoch hat sich der obere Teil von der Naht bis zur Klemme schwach mit Blut gefüllt. 
Versuch Nr. 76. V. saphena sin. 7. VI. 19r2 -. Dasselbe. Unter Beobachtung.

Welpe, $3,5 \mathrm{~kg}$ schwer. Versuch Nr. 77. Art. carotis sin. 12. VI. 1912 - zachiger Schnitt, mit Seide vernäht, blutet fast nicht, pulsiert.

Versuch Nr. 78. V.jugularis sin. I2. VI. I912- querer Schnitt mit zeitlicher Anlegung zweier durchgehender fixierender Ligaturfäden. Nachdem die Ligaturfäden durchschnitten und beicle Gefäßenden an ihnen auseinandergezogen waren, machte man längs des Gefäßes $\mathrm{I} 1 / 2 \mathrm{~mm}$ lange Mitteleinschnitte, so daß hiermit eine kreisförmige Schnittführung mit Längseinschnitten erhalten wurde. In den Lappenzwischenräumen an den Enden der Längseinschnitte wurden zwei Grundnähte angelegt. Seidennaht, nach Entfernen der Klemmen hat sich die Vene gut mit Blut gefüllt, in der Nahtstelle scheint das Gefäß auf Augenmaß breiter zu sein als an den übrigen Stellen. Ganz geringe Blutung.

Versuch Nr.79. V. femoralis sin. 19. VI. I912 -schräger Schnitt mit durchgehenden fixierenden Ligaturen, mit Seide vernäht, hat sich gut mit Blut gefüllt.

Versuch Nr. 8o. V. femoralis dextr. I9. VI. IgIz querer Schnitt mit durchgehenden fixierenden Ligaturen und Längrseinschnitten. Seidennaht, Blutung gering, die Nahtstelle ist auf Augenmaß breiter als das übrige Gefäß

Das Hündchen ist zur dauernden Beobachtung geblieben.

Ziemlich großerschwarzer Hund. Versuch Nr. 8 I. V. saphena dextr. Querer Schnitt mit zwei durchgehenden fixierenden Ligaturen. $\mathrm{Da}$ das Gefäß aus der Klemme glitt, riß am oberen Ende die cine Ligatur aus und man war gezwungen, das Gefäß noch cinmal einzuklemmen. Das Auffinden des Gefäßlumens war hier sehr schwierig und es ist gefunden worden an Hand der einen gebliebenen Ligatur. Die Naht war sehr schwierig, es entstand eine leichte Verengerung.

Versuch Nr. 82. V. femoralis dextr. I4. VI. I9I2 schräger Schnitt mit zwei durchgehenden fixierenden Ligaturen; Seidennaht, keinerlei Verengerung, das Gefäß hat sich mit Blut gut ausgedehnt. Der II und unter Beobachtung.

Auf Grund der oben angeführten Beobachtungen kann man folgende Schlüsse ziehen.

I. Die Veränderung der Richtung der Gefäßnahtlinie aus der queren in eine schräge und die durch Anwendung zackiger, lappenförmiger und kreisförmiger Schnittführungen mit Längseinschnitten komplizierteren Nahtformen stellen eigentlich für das Gefäß gar keine Gefahr vor. 
2. Die Technik einer Nahtanlegung bei schrägem Schnitt unterscheidet sich von der allgemeinen Carrel-Stichschen Methode nur durch Anwendung zweier Grundnähte.

3. Die Technik der Nahtanlegung bei übrigem Schnitt wird durch Entfernen der freien Lappenränder von der Mitte des Gefäßes erleichtert, was die Möglichkeit gibt, bei zwei Grundnähten zu nähen, ohne dabei Gefahr zu laufen, die gegenüberliegende Gefäßwand mitzufassen.

4. Vorteile der oben angeführten Methode bei ihrer richtigen Anwendung sind - die ausgeschlossene Möglichkeit einer zirkulären Gefäßverengerung bei schräger Schnittführung und selbst eine Erweiterung der Gefäßrinne, wie das die in den Protokollen angeführten Messungen des Gefäßumfanges bei den übrigen Schnittführungen zeigen.

5. Die im gegebenen Falle erhaltene Erweiterung des Gefäßlumens ist kein Aneurysma, das eine pathologische Veränderung in den Gefälgeweben voraussetzt, sondern ist durch eine geringe Lageveränderung der Gefäßwand gebildet und hat, nach den Resultaten der Beobachtungen zu urteilen, im Laufe der Zeit die Tendenz, vollständig zu verschwinden. In letzter Hinsicht sind weitere Beobachtungen und detaillierteres Studium der dabei stattfindenden Prozesse nötig.

6. Das zeitweise Anlegen fixierender Durchgangsligaturen bei der Venennaht erleichtert außerordentlich das eigentliche Anlegen der Naht selbst; gibt dabei die Möglichkeit, sie besonders vorsichtig zu machen und geht in Kombination mit den verschiedenen Schnittformen der Möglichkeit einer Gefäßlumenverengerung wesentlich aus dem Wege.

Zum Schluß spreche ich dem Herrn Chef der pathologischen Abteilung des kaiserl. Instituts für experimentelle Medizin Lfim Semjonowitsch London für dic Ćberlassung des Themas und die beständige Hilfe bei deren Ausarbeitung meinen besonders innigen Dank aus. 


\section{Erklärung der Abbildungen auf Tafel II.}

r. Versuch Nr. 4I, schräger Schnitt, Art. femoralis, 4. Tag nach der Operation.

2. Versuch Nr. 70, schräger Schnitt, V. jugularis ext., 21. Tag nach der Operation.

3. Versuch Nr. 5I, schräger Schnitt, Art. femoralis, 37. Tag nach der Operation.

4. Versuch Nr. 50, schräger Schnitt, V. jugularis d., 5I. Tag nach der Operation.

5. Versuch Nr. 39, zackiger Schnitt, Art. carotis, 4. Tag nach der Operation.

6. Versuch Nr. 29, zackiger Schnitt, Art. carotis, 5. Tag nach der Operation.

7. Versuch Nr. 1o, zackiger Schnitt, Art. femoralis, II. Tag nach der Operation.

8. Versuch Nr. 42, zackiger Schnitt, Art. carotis sin., 85. Tag nach der Operation.

9. Versuch Nr. 40, kreisförmiger Schnitt mit Längseinschnitten. Art. carotis, 4. Tag nach der Operation.

1o. Versuch Nr. 36, kreisförmiger Schnitt mit Längseinschnitten, Art. carotis, 33. Tag nach der Operation.

I I. Versuch Nr. 53, kreisförmiger Schnitt mit Längseinschnitten, Art. carotis, 37. Tag nach der Operation.

12. Versuch Nr. 43, kreisförmiger Schnitt mit Längseinschnitten, Art. carotis i., 85. Tag nach der Operation.

13. Versuch Nr. 61, Lappenschnitt, Art. carotis s., Haarnaht, 35. Tag nach der Operation.

14. Versuch Nr. 60, Lappenschnitt, Art. carotis d., Haarnaht, 35. Tag nach der Operation.

15. Versuch Nr. 67, Querschnitt, V. femoralis d., 35. Tag nach der Operation. I6. Versuch Nr. 68, Lappenschnitt, V. femoralis s., 35. Tag nach der Operation.

\section{Literaturverzeichnis.}

I. Bertram, M. Bernheim, Arteriovenous anastomosis - reversal of the circulation - as a preventive of gangrene of the extremities. Annals of surgery 1912, Vol. LV, p. 195.

2. B orst u. Enderlen, Über Transplantation von Gefäßen und ganzen Organen. Deutsche Zeitschr. f. Chir. I909, Bd. 99, S. 54.

3. Carre1, La téchnique opératoire des anastomoses vasculaires et la transplantation des viscères. Lyon médical igo2, t. XCVIII, p. 859.

4. Derselbe, Exstirpation et réplantation de la glande thyroide avec réversion de la circulation. C. R. de la Soc. de Biol. I905.

5. Derselbe, Results of the transplantation of blood vessels, organs and limbs. The Journal of the Americ. medic. Assoc. 1908, November, Vol. LI. 


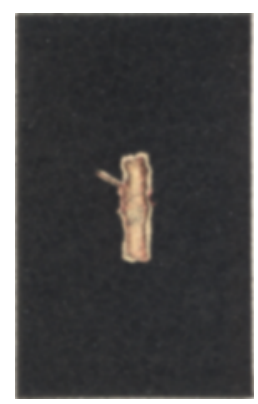

1.

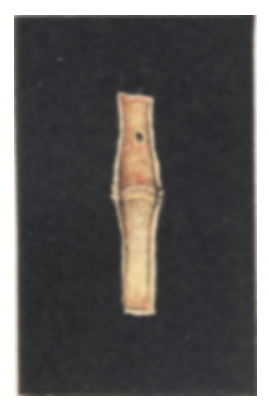

5.

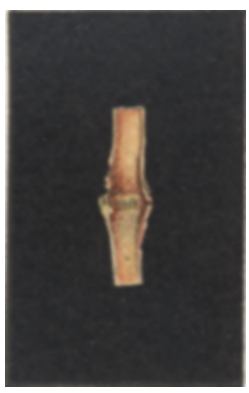

9.

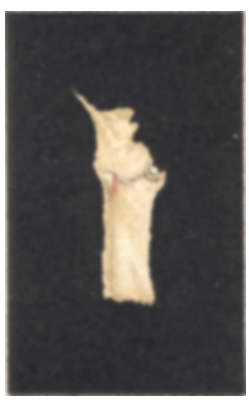

13.

Dobrowolskaja.

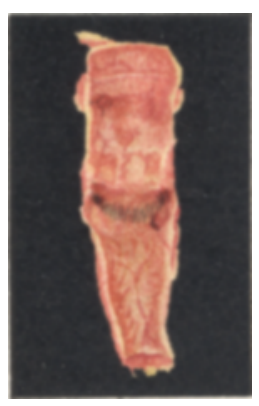

2.

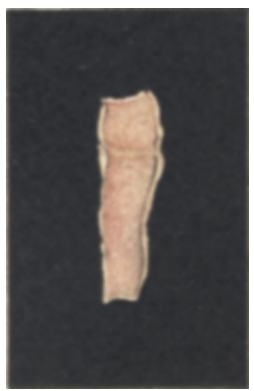

6.

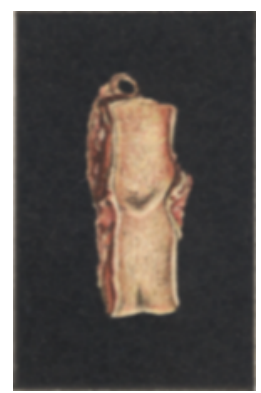

10.

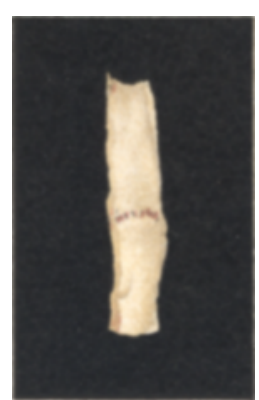

14.

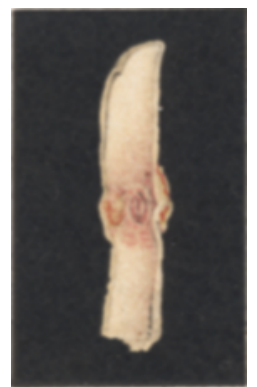

11.

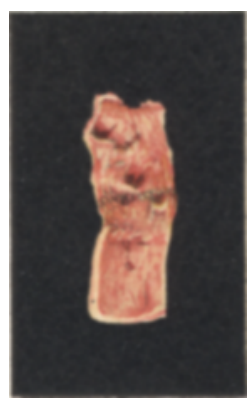

15.

Vorlag von F.C.W.Vogel in Leipzig.

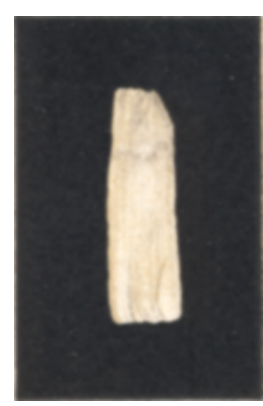

4.

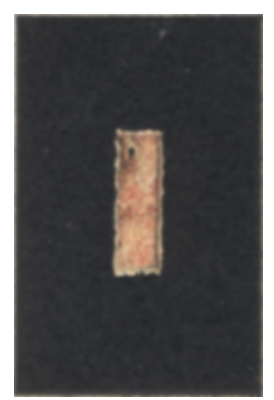

8.

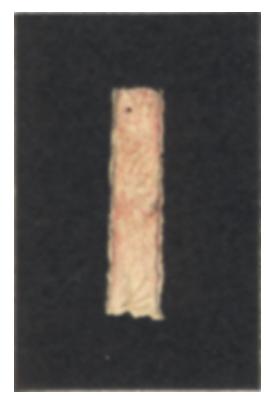

12.

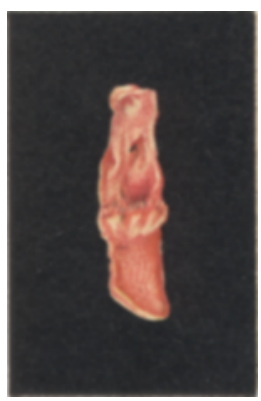

16.

Lith Anst v. Johames Amdt, Jena. 
6. Carrel, La transplantation des membres. Revue de chirurgie I908, t. 38, p. 672 .

7. Derselbe, Joppelte Nephrektomic und Reimplantation einer Niere. Arch. f. klin. Chir. Ig09, Bd. 88, S. 379.

8. Derselbe and Guthrie, Transplantation of blood vessels and or. gans. Brit. med. Journ. 1906, 22. Dezember.

9. Dieselben, De la transplantation uniterminal des veines sur les artères. C. R. de la Soc. de Biol. 1905, t. 59, p. 596.

10. Dieselben, The reversal of the circulation in a limb. Annals of surgery 1906, February.

II. Chalmersda Costa, Operation for aneurismal varix of the popliteal vessels. Annals of surgery 1912, Vol. LV, p. 593.

12. De l b e t. Bullet. médic. Igo6, p. I I I9.

13. Enderlen, Untersuchungen über die Transplantation der Schilddrüse in die Bauchhöhle von Katzen und IIunden. Mitteil, a. d. Grenzgeb. I 898, Bd. 3 .

14. Derselbe u. Borst, Beiträge zur Gefäßchirurgie und zur Organtransplantation. Münchn. med. Wochenschr. 1910, Nr. 36 .

15. Garre, Uber Gefäß- und Organtransplantation. Deutsche med. Wochenschrift 1909, Nr. 40, S. 1735 .

16. Guthrie, Transplantation of Organs. Journal of Americ medic. Assoc. 1910, 12. March.

17. Derselbe, Suture of blood vessels with human hair. The Journ. of Americ. medic. Assoc. I9ro, Vol. LIV, p. 349.

18. Jaboulay, Tentatives de greffe d'un rein d'animal chez l'homme. La semaine médic. 1906, Nr. 40, p. 473.

19. J en sen, ëber zirkuläre Gefäßsutur. Arch. f. klin. Chir. I903, Bd. 69, S. 938 .

20. I. is s ow skaja, Zur Transplantation der Schilddrüse. Dissertat. St. Pctersburg $19 \mathrm{II}$.

21. Lo ndon, I. S., Die Gefäßnaht und Massen-Transplantationen. Handbuch der biochem. Arbeitsmethoden 1912, Bd. 5, S. 815.

22. M a $t$ as, The statistics of endoaneurysmorrhaphy or the radical cure of ancurysm by intrasaccular suture. Journ. of the Americ. medic. Assoc. 1908, I 4. Nov.

23. Morozowa, Zur L.ehre von der Gefäßnaht. Dissertat. St. Petersburg 1909.

24. Murphy, Arteriennaht. The Lancet Igog.

25. Payr, Beiträge zur Technik der Blutgefäße und Nervennaht. Arch. f. klin. Chir., Bd. 62.

26. I) ers clbe, Weitere Mitteilungen über Verwendung des Magnesium bei der Naht der Blutgefäße. Arch. f. klin. Chir., Bd. 64 .

27. P'i r ovano, Nouveau procédé de suture artérielle et veineuse. Revue de Chir. I909, t. 40, p. 669.

28. Ru ot te. Lyon médic. 1907, Nr. 40. 
54 Dobrowolskaja, Zur Technik der Nähte an GefäBen kleinen Kalibers.

29. Schack u. Hesse. Russki Wratsch I9II, Nr. 6, 8 u. 9 .

3o. S of ot e r of $f$, Zur Gefäßnaht. Dissertat. Tomsk rolo (russisch).

3r. Derselbe, Frauenhaar als Material für die Gefäßnaht. Zentralbl.

f. Chir. I9I I, Nr. 2 I.

32. S $\mathrm{t}$ i $\mathrm{ch}$, Zur Transplantation von Organen mittels Gefäßnaht. Verhandl. d. Deutsch. Gesellsch., 36. Kongreß 1907, S. Io.

33. Derselbe u. Makkas, Zur Transplantation der Schilddrüse mittels Gefäßnaht. Beitr. z. klin. Chir., Bd. 60, S. $43 \mathrm{I}$.

34. Dieselben u. Dowman, Beiträge zur Gefäßchirurgie. Beitr. z. klin. Chir. I907, Bd. 53, S. I I3.

35. Ung er. Zentralbl. f. Chir. I909, S. I I4.

36. W i et ing, Die angiosklerotische Gangrän und ihre operative Behandlung durch arterio-venöse Intubation. Deutsche med. Wochenschr. I908, Nr. 28.

37. Derselbe, Die angiosklerotische Gangrän und ihre operative Behandlung durch Überleitung des arteriellen Blutstroms in das Venensystem. Deutsche Zeitschr. f. Chir. I9II, Bd. I ro, S. 364.

38. Y a ma no ü chi, H., Über die zirkulären Gefäßnähte und Arterienvenenanastomosen, sowie über die Gefäßtransplantationen. Deutsche Zeitschr. f. Chir. I9I I, Bd. I 2.

39. Z e id l er. Russisch. Arch. f. Chir. 1910, Bd. 26. 